\title{
Degeneration of Solitons for a the $(3+1)$ - dimensional Generalized Nonlinear Evolution Equation for the Shallow Water Waves
}

longxing li ( $\square$ llxyz891008@163.com )

Qujing Normal University https://orcid.org/0000-0003-2141-4475

Long-Xing Li

Qujing Normal University

\section{Research Article}

Keywords: Shallow-water waves, T-breather, M-lump, Hybrid solution

Posted Date: December 20th, 2021

DOI: https://doi.org/10.21203/rs.3.rs-1179230/v1

License: (c) (1) This work is licensed under a Creative Commons Attribution 4.0 International License.

Read Full License

Version of Record: A version of this preprint was published at Nonlinear Dynamics on February 14th, 2022. See the published version at https://doi.org/10.1007/s11071-022-07270-4. 


\title{
Degeneration of solitons for a the (3+1)-dimensional generalized nonlinear evolution equation for the shallow water waves
}

\author{
Long-Xing Li \\ College of Mathematics and Statistics, Qujing Normal University, Qujing, Yunnan 655000, China
}

\begin{abstract}
A the (3+1)-dimensional generalized nonlinear evolution equation for the shallow water waves is investigated with different methods. Based on symbolic computation and Hirota bilinear form, $\mathrm{N}$ soliton solutions are constructed. In the process of degeneration of $N$-soliton solutions, $T$-breathers are derived by taking complexication method. Then rogue waves will emerge during the degeneration of breathers by taking the parameter limit method. Through full degeneration of $N$-soliton, $M$-lump solutions are derived based on long wave limit approach. In addition, we also find out that the partial degeneration of $\mathrm{N}$-soliton process can generate the hybrid solutions composed of soliton, breather and lump.
\end{abstract}

Keywords Shallow-water waves. $T$-breather. $M$-lump Hybrid solution

\section{Introduction}

Shallow water waves refer to the water waves with large water wavelength relative to the depth [1], also called the long water waves. There are some natural problems are related to the shallow water waves, such as the tides, storms, atmospheric flows and tsunamis [2,3]. Nonlinear evolution equations (NLEEs) describe lots of physical and science phenomena, are fundamental models in nonlinear science, especially in nonlinear dynamics. The wave phenomenon of NLEEs are playing an essential role in studying various areas of physics and mathematics, such as nonlinear optics, plasmas, oceanography and Bose-Einstein condensates [4-9]. Solitons, breathers, lumps and rogue waves are typical wave models to study nonlinear scientific issues $[10,11,12]$. Lump wave is a special kind of rational function wave with the characteristics of energy concentration and localization property in the space [13-18]. In the train of the study, a series of methods has been quickly developed to obtain lump wave including the first mathematical description of lump wave [19], the long wave limit method is an effective method proposed to study the $M$-lump solutions of the integrable systems in [20-24], this positive method has attracted a lot of researchers' attention. the direct method [25-30], the parameter limit method [31-34], complexication method [35,36], bilinear neural network method [37], etc. Through these methods, so many valuable and interesting results have sprung e-mail: llxyz891008@163.com (corresponding author) 
up. Inspired by the previous studies, we mainly focus on the $(3+1)$-dimensional generalized nonlinear evolution equation for the shallow water waves to study the evolution and degeneration behaviour of $N$-solitons in this work. By applying the Horita bilinear method [34] and other systematic method, the structures of solutions for this equation are analyzed.

In this paper, we mainly investigate the $(3+1)$-dimensional generalized nonlinear evolution equation for the shallow water waves $[35,36]$, the equation reads

$$
l_{1} u_{x z}+\left(l_{2} u_{t}+l_{3} u_{x x x}+l_{4} u u_{x}\right)_{y}+l_{5}\left(u_{x} \partial_{x}^{-1} u_{y}\right)_{x}=0
$$

where $u(x, y, z, t)$ is a real differential function of the independent variables $x, y, z$ and $t, \partial_{x}^{-1}$ represents the integral with respect to $x, l_{i}(i=1,2,3,4,5)$ are real nonzero constants. Eq.(1) is also a KdV-type equation which can be regard as a model for the incompressible [37].

Based on the previous researches, we mainly investigate the deformation behaviors of $N$-soliton, most of solutions solutions obtained for Eq.(1) are new and may be very meaningful to explain some special nonlinear science phenomenon. In Section 2, $N$-soliton solution for Eq.(1) is given and $T$-breathers are derived. $M$-lumps are obtained by long wave limit method in Section 3. Hybrid solutions consisted of solitons and breathers or lumps are obtained in Section 4. A brief conclusion is given in Section 5 finally.

\section{From $N$-soliton solutions to $T$-order breather solutions}

In the following, we will obtain $N$-soliton solutions for Eq.(1) by Hirota bilinear method, and according to taking the conjugate complex of the parameters of the $N$-soliton $(N=2 T)$ solutions, $T$-breather solutions also can be obtained. Firstly, we take the transformation

$$
u(x, y, z, t)=\frac{6 l_{3}}{l_{4}}(\ln f)_{x x}
$$

and

$$
l_{4}=l_{5},
$$

where $f$ is an unknown function about $x, y, z, t$. Eq.(1) can be converted to the bilinear operator $D$ as follows

$$
\left(l_{1} D_{x} D_{z}+l_{2} D_{y} D_{t}+l_{3} D_{x}^{3} D_{y}\right) f \cdot f=0
$$

The Hirota bilinear operator $D_{x}^{m} D_{t}^{n}$ are defined by [31] $(n, m \geq 0)$.

$D_{x}^{l} D_{y}^{m} D_{z}^{n} D_{t}^{r} f(x, t) \cdot g(x, t)=\left.\left(\frac{\partial}{\partial x}-\frac{\partial}{\partial x^{\prime}}\right)^{l}\left(\frac{\partial}{\partial y}-\frac{\partial}{\partial y^{\prime}}\right)^{m}\left(\frac{\partial}{\partial z}-\frac{\partial}{\partial z^{\prime}}\right)^{n}\left(\frac{\partial}{\partial t}-\frac{\partial}{\partial t^{\prime}}\right)^{r} f(x, y, z, t) g\left(x^{\prime}, y^{\prime}, z^{\prime}, t^{\prime}\right)\right|_{x^{\prime}=x, y^{\prime}=y, z^{\prime}=z, t^{\prime}=t}$.

On the basis of $[30,31]$, we choose the following test functions

$$
f=f_{N}=\sum_{\mu=0,1} \exp \left(\sum_{i=1}^{N} \mu_{i} \eta_{i}+\sum_{i<j}^{N} A_{i j} \mu_{i} \mu_{j}\right)
$$


where $\eta_{i}=k_{i}\left(x+p_{i} y+q_{i} y+r_{i} t\right)+\eta_{i}^{(0)}, k_{i} \neq 0, p_{i} \neq 0, q_{i} \neq 0, \eta_{i}^{(0)}(i=1,2,3, \cdots)$ are arbitrary real constants. The first $\sum$ is the summation of all possible combinations of $\mu_{1}=0,1, \mu_{2}=0,1, \cdots$, $\mu_{N}=0,1$. Another $\sum$ means over all possible combinations of the $N$ parts with the particular form $i<j$. According to Eq.(3) and (4), we get

$$
\left\{\begin{array}{l}
r_{i}=\frac{-l_{3} k_{i}^{2} p_{i}-l_{1} q_{i}}{l_{2} p_{i}}, \\
e^{A_{i j}}=\frac{-3 l_{3} k_{i} k_{j}\left(p_{i}+p_{j}\right)+l_{3} k_{i}^{2}\left(2 p_{i}+p_{j}\right)+l_{3} k_{j}^{2}\left(p_{i}+2 p_{j}\right)-l_{2}\left(p_{i}-p_{j}\right)\left(r_{i}-r_{j}\right)}{3 l_{3} k_{i} k_{j}\left(p_{i}+p_{j}\right)+l_{3} k_{i}^{2}\left(2 p_{i}+p_{j}\right)+l_{3} k_{j}^{2}\left(p_{i}+2 p_{j}\right)-l_{2}\left(p_{i}-p_{j}\right)\left(r_{i}-r_{j}\right)} .
\end{array}\right.
$$

Here $N$ is a positive integer, the positive constant $e^{A_{i j}}$ is not necessary component of the soliton, but it is very convenient to the calculation of breathers.

Generally, the function expressed in Eq.(4) can not generate a breather solution of Eq.(1) if only one of above constants takes a complex number. However, when taking the paired complex value of above real parameters, smooth breathers of Eq.(1) can be yielded on a zero background. This idea named the paired complex valuation of solitons has a long history which goes back to the works for few decades ago $[6,8,9]$. We also note that there are some constraints on $e^{A_{i j}}$ to guarantee reality and smoothness of the breathers generated by the expression in Eq.(4).

Based on the idea of taking complex value mentioned above, we take

$$
\left\{\begin{array}{l}
k_{2 j-1}=k_{j 1}+i k_{j 2}, k_{2 j}=k_{j 1}-i k_{j 2}, \\
p_{2 j-1}=p_{j 1}+i p_{j 2}, p_{2 j}=p_{j 1}-i p_{j 2}, \\
q_{2 j-1}=q_{j 1}+i q_{j 2}, q_{2 j}=q_{j 1}-i q_{j 2}, \\
\eta_{j}^{(0)}=0, j=1,2, \cdots, \frac{N}{2} .
\end{array}\right.
$$

$k_{j 1}, k_{j 2}, p_{j 1}, p_{j 2}, q_{j 1}, q_{j 2}$ are real constants and $N$ is even. Now Eq.(4) will be rewritten as

$$
f=f_{2 T}=\sum_{\mu=0,1} \exp \left(\sum_{i=1}^{2 T} \mu_{i} \eta_{i}+\sum_{i<j}^{2 T} A_{i j} \mu_{i} \mu_{j}\right)
$$

Inserting Eq.(6) into Eq.(7), then

$$
\begin{aligned}
& \eta_{2 T-1}=\eta_{2 T}^{*}=\eta_{j 1}+i \eta_{j 2}, \\
& \eta_{j 1}=k_{j 1} x+\left(k_{j 1} p_{j 1}-k_{j 2} p_{j 2}\right) y+\left(k_{j 1} q_{j 1}-k_{j 2} q_{j 2}\right) z+\left(\frac{l_{3}}{l_{2}} k_{j 1}\left(3 k_{j 2}^{2}-k_{j 1}^{2}\right)+\frac{l_{1}}{l_{2}} \frac{k_{j 2}\left(p_{j 1} q_{j 2}-p_{j 2} q_{j 1}\right)-k_{j 1}\left(p_{j 1} q_{j 1}+p_{j 2} q_{j 2}\right)}{p_{j 1}^{2}+p_{j 2}^{2}}\right) t, \\
& \eta_{j 2}=k_{j 2} x+\left(k_{j 2} p_{j 1}+k_{j 1} p_{j 2}\right) y+\left(k_{j 2} q_{j 1}+k_{j 1} q_{j 2}\right) z+\left(\frac{l_{3}}{l_{2}} k_{j 2}\left(k_{j 2}^{2}-3 k_{j 1}^{2}\right)+\frac{l_{1}}{l_{2}} \frac{k_{j 1}\left(p_{j 2} q_{j 1}-p_{j 1} q_{j 2}\right)-k_{j 2}\left(p_{j 1} q_{j 1}+p_{j 2} q_{j 2}\right)}{p_{j 1}^{2}+p_{j 2}^{2}}\right) t, \\
& j=1,2, \cdots, T .
\end{aligned}
$$

Case 1. $\mathbf{T}=\mathbf{1}$ A 1 -order breather $u_{b}^{[1]}=\frac{6 l_{3}}{l_{4}}\left(\ln f_{b}^{[1]}\right)_{x x}$ is constructed by

$$
f_{b}^{[1]}=1+2 e^{\eta_{11}} \cos \left(\eta_{12}\right)+e^{A_{12}} e^{2 \eta_{11}}
$$

and

$$
e^{A_{12}}=\frac{3 l_{3} k_{12}^{2} p_{11}\left(p_{11}^{2}+p_{12}^{2}\right)+l_{3} k_{11} k_{12} p_{12}\left(p_{11}^{2}+p_{12}^{2}\right)+l_{1} p_{12}\left(p_{11} q_{12}-p_{12} q_{11}\right)}{-3 l_{3} k_{11}^{2} p_{11}\left(p_{11}^{2}+p_{12}^{2}\right)+l_{3} k_{11} k_{12} p_{12}\left(p_{11}^{2}+p_{12}^{2}\right)+l_{1} p_{12}\left(p_{11} q_{12}-p_{12} q_{11}\right)}>0
$$


$\eta_{11}$ and $\eta_{12}$ are real component and imaginary component of $\eta_{1}$ respectively. For a given time $t$, the trajectory of 1-order breather is a line $l_{1}$ on $(x, y)$-plane, $l_{1}: \eta_{11}=k_{11} x+\left(k_{11} p_{11}-k_{12} p_{12}\right) y+\left(k_{11} q_{11}-\right.$ $\left.k_{12} q_{12}\right) z+\left(\frac{l_{3}}{l_{2}} k_{11}\left(3 k_{12}^{2}-k_{11}^{2}\right)+\frac{l_{1}}{l_{2}} \frac{k_{12}\left(p_{11} q_{12}-p_{12} q_{11}\right)-k_{11}\left(p_{11} q_{11}+p_{12} q_{12}\right)}{p_{11}^{2}+p_{12}^{2}}\right) t=0$, and periodic along the direction of the line $l_{2}$ on $(x, y)$-plane, $l_{2}: \eta_{12}=k_{12} x+\left(k_{12} p_{11}+k_{11} p_{12}\right) y+\left(k_{12} q_{11}+k_{11} q_{12}\right) z+\left(\frac{l_{3}}{l_{2}} k_{12}\left(k_{12}^{2}-3 k_{11}^{2}\right)+\right.$ $\left.\frac{l_{1}}{l_{2}} \frac{k_{11}\left(p_{12} q_{11}-p_{11} q_{12}\right)-k_{12}\left(p_{11} q_{11}+p_{12} q_{12}\right)}{p_{11}^{2}+p_{12}^{2}}\right) t=0$. That is to say, the location depends on $k_{11}$ and $k_{11} p_{11}-k_{12} p_{12}$, the period depends on $k_{12}$ and $k_{12} p_{11}+k_{11} p_{12}$ on $(x, y)$-plane. Three forms of 1-order breather for Eq.(1) are constructed with different values of $k_{11}, k_{12}, p_{11}$ and $p_{12}$ in Eq.(9). When taking $k_{11}=0$ and $k_{11} p_{11}-k_{12} p_{12} \neq 0$, Fig.1(a) displays the 1-order breathers are parallel to the $x$-axis on the $(x, y)$-plane as $k_{1}=\frac{1}{6} i, k_{2}=-\frac{1}{6} i, p_{1}=\frac{1}{4}+i, p_{2}=\frac{1}{4}-i, q_{1}=\frac{1}{10}+i, q_{2}=\frac{1}{10}-i$. When taking $k_{11} \neq 0$ and $k_{11} p_{11}-k_{12} p_{12}=0, k_{1}=\frac{1}{4}+i, k_{2}=\frac{1}{4}-i, p_{1}=\frac{1}{6}+\frac{1}{24} i, p_{2}=\frac{1}{6}-\frac{1}{24} i, q_{1}=\frac{1}{10}+i, q_{2}=\frac{1}{10}-i$, the 1 -order breathers are parallel to the $y$-axis shown in Fig.1(b). When taking $k_{11} \neq 0$ and $k_{11} p_{11}-k_{12} p_{12} \neq 0$, the 1-order breathers are parallel to the the line $k_{11} x+\left(k_{11} p_{11}-k_{12} p_{12}\right) y=0$ shown in Fig.1(c) with $k_{1}=\frac{1}{4}+i, k_{2}=\frac{1}{4}-i, p_{1}=\frac{1}{6}+\frac{1}{6} i, p_{2}=\frac{1}{6}-\frac{1}{6} i, q_{1}=\frac{1}{10}+i, q_{2}=\frac{1}{10}-i$.
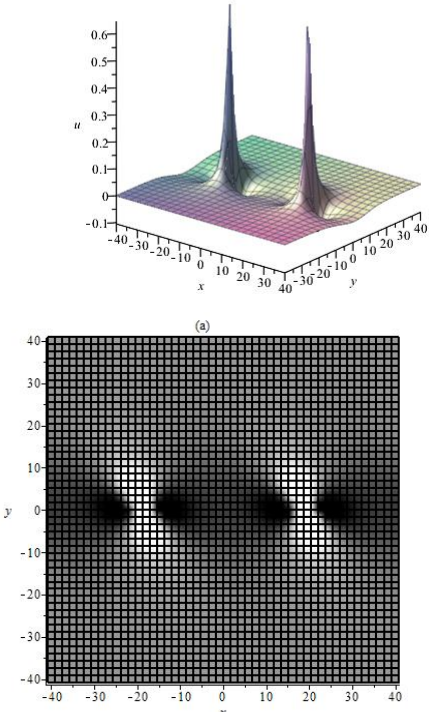
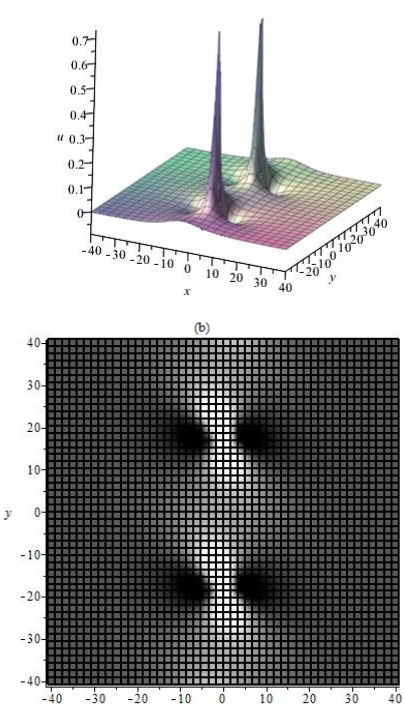
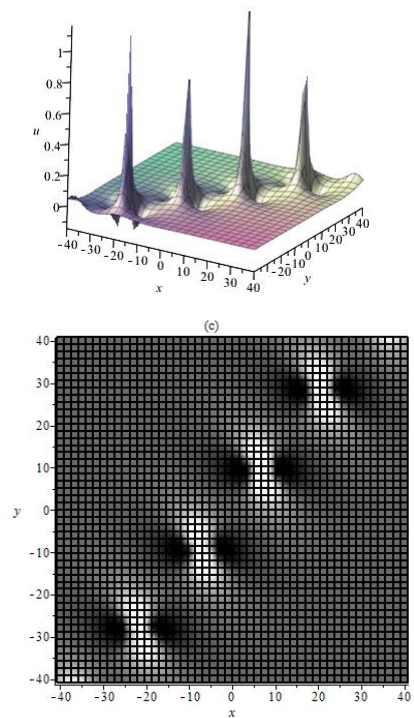

Fig. 1: Different spatial structures of 1-order breather $u_{b}^{[1]}$ as $l_{1}=2, l_{2}=1, l_{3}=1, l_{4}=3, z=0, t=0$. 
Case 2. $\mathbf{T}=\mathbf{2}$ A 2-order breather $u_{b}^{[2]}=\frac{6 l_{3}}{l_{4}}\left(\ln f_{b}^{[2]}\right)_{x x}$ is constructed by

$$
\begin{aligned}
f_{b}^{[2]}= & 1+e^{\eta_{1}}+e^{\eta_{2}}+e^{\eta_{3}}+e^{\eta_{4}}+e^{A_{12}} e^{\eta_{1}+\eta_{2}}+e^{A_{13}} e^{\eta_{1}+\eta_{3}}+e^{A_{14}} e^{\eta_{1}+\eta_{4}}+e^{A_{23}} e^{\eta_{2}+\eta_{3}}+e^{A_{24}} e^{\eta_{2}+\eta_{4}}+e^{A_{34}} e^{\eta_{3}+\eta_{4}} \\
& +e^{A_{12}} e^{A_{13}} e^{A_{23}} e^{\eta_{1}+\eta_{2}+\eta_{3}}+e^{A_{12}} e^{A_{14}} e^{A_{24}} e^{\eta_{1}+\eta_{2}+\eta_{4}}+e^{A_{13}} e^{A_{14}} e^{A_{34}} e^{\eta_{1}+\eta_{3}+\eta_{4}}+e^{A_{23}} e^{A_{24}} e^{A_{34}} e^{\eta_{2}+\eta_{3}+\eta_{4}} \\
& +e^{A_{12}} e^{A_{13}} e^{A_{14}} e^{A_{23}} e^{A_{24}} e^{A_{34}} e^{\eta_{1}+\eta_{2}+\eta_{3}+\eta_{4}}
\end{aligned}
$$

with $\eta_{1}=\eta_{2}^{*}=\eta_{11}+\eta_{12} i, \eta_{3}=\eta_{4}^{*}=\eta_{21}+\eta_{22} i, e^{A_{i j}}=\frac{-3 l_{3} k_{i} k_{j}\left(p_{i}+p_{j}\right)+l_{3} k_{i}^{2}\left(2 p_{i}+p_{j}\right)+l_{3} k_{j}^{2}\left(p_{i}+2 p_{j}\right)-l_{2}\left(p_{i}-p_{j}\right)\left(r_{i}-r_{j}\right)}{3 l_{3} k_{i} k_{j}\left(p_{i}+p_{j}\right)+l_{3} k_{i}^{2}\left(2 p_{i}+p_{j}\right)+l_{3} k_{j}^{2}\left(p_{i}+2 p_{j}\right)-l_{2}\left(p_{i}-p_{j}\right)\left(r_{i}-r_{j}\right)}$, $r_{i}=\frac{-l_{3} k_{i}^{2} p_{i}-l_{1} q_{i}}{l_{2} p_{i}}$, and the definitions in Eq.(6) and Eq.(8). When $\eta_{1} \neq \eta_{3}, l_{1}=2, l_{2}=1, l_{3}=1, l_{4}=$ $3, q_{1}=q_{2}=q_{3}=q_{4}=-3, z=0, t=0$, the breathers are parallel pattern (Fig.2(a)), and the intersectant pattern is shown in Fig.2(b). When $\eta_{1}=\eta_{3}$, the breather degenerates to 1-order breather (Fig.2(c)).
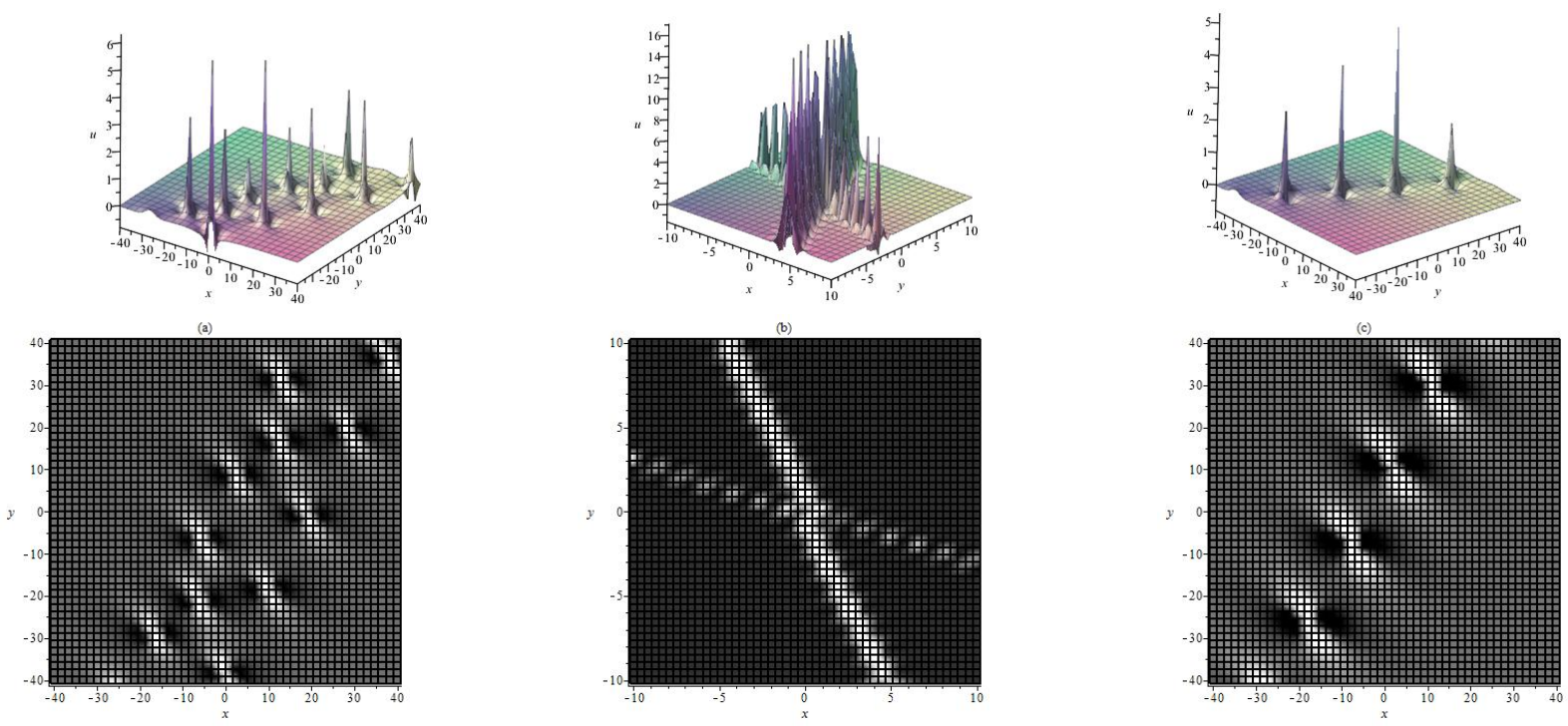

Fig. 2: The 2-order breather $u_{b}^{[2]}$ as (a) parallel pattern with $k_{1}=\frac{1}{6}+\frac{1}{6} i, k_{2}=\frac{1}{6}-\frac{1}{6} i, k_{3}=\frac{1}{4}+\frac{1}{4} i, k_{4}=$ $\frac{1}{4}-\frac{1}{4} i, p_{1}=\frac{1}{2}+i, p_{2}=\frac{1}{2}-i, p_{3}=\frac{1}{2}+i, p_{4}=\frac{1}{2}-i,(\mathrm{~b})$ intersectant pattern with $k_{1}=1-2 i, k_{2}=1+2 i, k_{3}=$ $2-i, k_{4}=2+i, p_{1}=-2+\frac{5}{2} i, p_{2}=-2-\frac{5}{2} i, p_{3}=-\frac{1}{2}+2 i, p_{4}=-\frac{1}{2}-2 i$, (c) degenerated pattern with $k_{1}=\frac{1}{6}+\frac{1}{6} i, k_{2}=\frac{1}{6}-\frac{1}{6} i, k_{3}=\frac{1}{6}+\frac{1}{6} i, k_{4}=\frac{1}{6}-\frac{1}{6} i, p_{1}=\frac{1}{2}+i, p_{2}=\frac{1}{2}-i, p_{3}=\frac{1}{2}+i, p_{4}=\frac{1}{2}-i$.

Case 3. $\mathbf{T}=\mathbf{3}$ The 3 -order breathers with different shapes are shown in Fig. 3 with $l_{1}=2, l_{2}=1, l_{3}=$ $1, l_{4}=3, q_{1}=q_{2}=q_{3}=q_{4}=-3, z=0, t=0$. In case of $\eta_{1} \neq \eta_{3} \neq \eta_{5}$. An intersectant pattern of breather is displayed in Fig.3(a). In case of $\eta_{1}=\eta_{3}=\eta_{5}, p_{1} \neq p_{3} \neq p_{5}$, a mixed pattern of breather is displayed in Fig.3(b); while a parallel pattern of breather with appropriate walue is displayed in Fig.3(c). In case of $\eta_{1}=\eta_{3}=\eta_{5}, p_{1}=p_{3}=p_{5}$, the 3-order breather degenerate to a 1-order breather completely 
(Fig.3(d)). From Fig.3, it means that the parameters in Eq.(6) affect and control the period, amplitude and phase of the breather, the interaction behaviours involved high-order breathers are elastic.
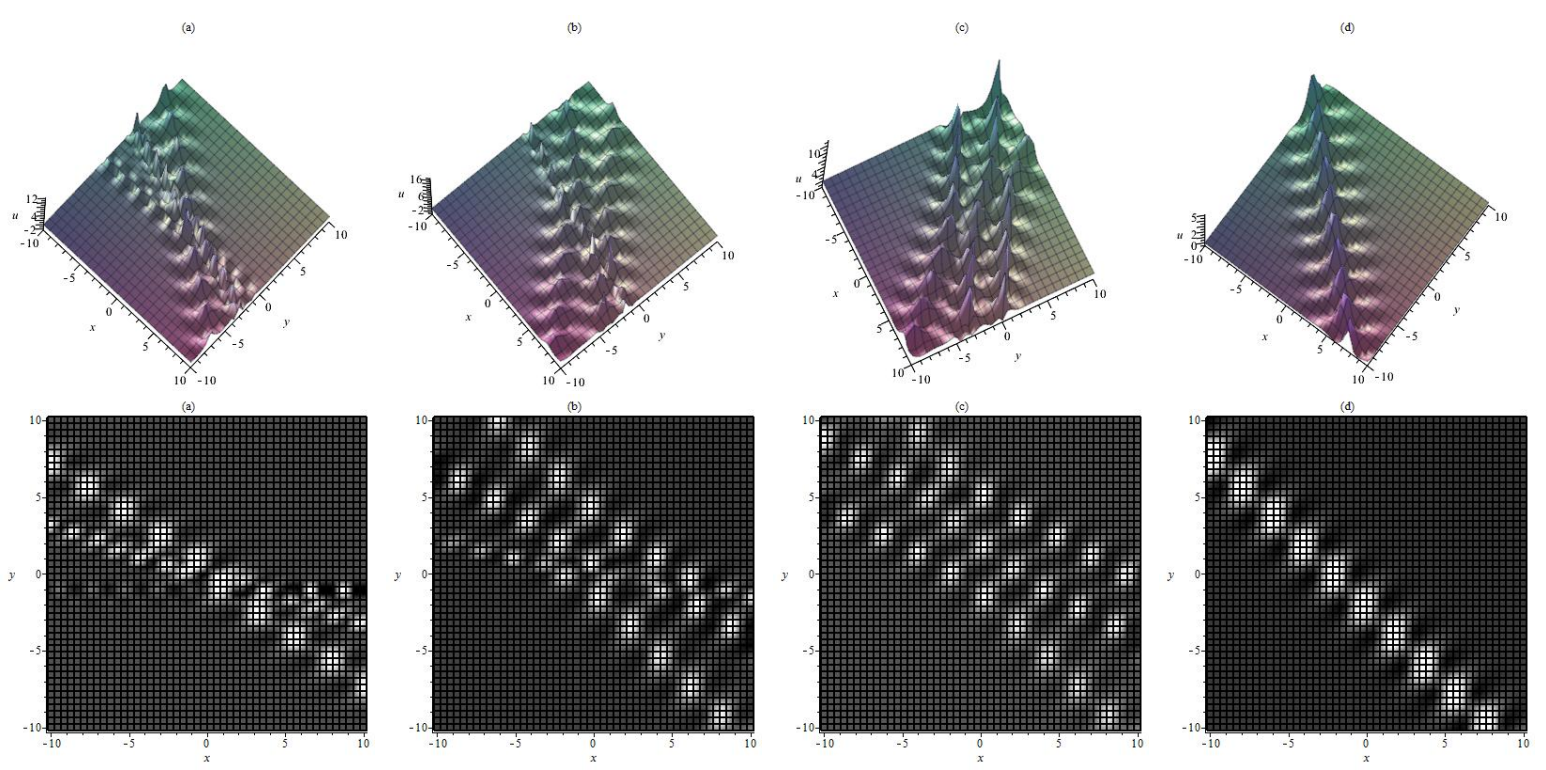

Fig. 3: The 3-order breather $u_{b}^{[3]}$ as (a) intersectant pattern with $k_{1}=1-2 i, k_{2}=1+2 i, k_{3}=1-i, k_{4}=1+i, k_{5}=$ $3 i, k_{6}=-3 i, p_{1}=-2+\frac{5}{2} i, p_{2}=-2-\frac{5}{2} i, p_{3}=-\frac{1}{2}+2 i, p_{4}=\frac{1}{2}-i, p_{5}=-5+i, p_{6}=-5-i$, (b) mixed pattern with $k_{1}=1-\frac{3}{2} i, k_{2}=1+\frac{3}{2} i, k_{3}=1-\frac{3}{2} i, k_{4}=1+\frac{3}{2} i, k_{5}=1-\frac{3}{2} i, k_{6}=1+\frac{3}{2} i, p_{1}=-\frac{1}{2}+i, p_{2}=-\frac{1}{2}-i, p_{3}=$ $-\frac{1}{2}+\frac{3}{2} i, p_{4}=-\frac{1}{2}-\frac{3}{2} i, p_{5}=-2+4 i, p_{6}=-2-4 i$, (c) parallel pattern with $k_{1}=1-\frac{3}{2} i, k_{2}=1+\frac{3}{2} i, k_{3}=1-\frac{3}{2} i, k_{4}=$ $1+\frac{3}{2} i, k_{5}=1-\frac{3}{2} i, k_{6}=1+\frac{3}{2} i, p_{1}=-\frac{1}{2}+i, p_{2}=-\frac{1}{2}-i, p_{3}=-\frac{1}{2}+\frac{3}{2} i, p_{4}=-\frac{1}{2}-\frac{3}{2} i, p_{5}=-\frac{1}{2}+\frac{7}{4} i, p_{6}=-\frac{1}{2}-\frac{7}{4} i$, (d) degenerated pattern with $k_{1}=1-\frac{3}{2} i, k_{2}=1+\frac{3}{2} i, k_{3}=1-\frac{3}{2} i, k_{4}=1+\frac{3}{2} i, k_{5}=1-\frac{3}{2} i, k_{6}=1+\frac{3}{2} i, p_{1}=$ $-\frac{1}{2}+i, p_{2}=-\frac{1}{2}-i, p_{3}=-\frac{1}{2}+i, p_{4}=-\frac{1}{2}-i, p_{5}=-\frac{1}{2}+i, p_{6}=-\frac{1}{2}-i$.

\section{Evolution and degeneration from soliton to Lump solution}

It is known that rogue wave or lump-type wave can be generated from breather by degeneration with parameter limit method $[12,24]$. By referring many studies, we also note that high-order lump can be generated in the process of degeneration of breather by long wave limit method. This section is consisted of two subsections, rogue wave or lump-type solution is obtained in subsection 3.1, $M$-lump is constructed in subsection 3.2 .

\subsection{Degeneration of breather and emergence of rogue wave or lump-type solution}

Under the bilinear form in Eq.(3), we choose a new test function which is first applied to Eq.(1)

$$
f(x, y, z, t)=\delta_{1} \cosh \phi_{1}+\delta_{2} \cos \phi_{2}+\delta_{3} \cosh \phi_{3}
$$

where $\phi_{i}=k_{i}\left(a_{i} x+b_{i} y+c_{i} z+d_{i} t+\gamma_{i}\right), k_{i}, a_{i}, b_{i}, c_{i}, d_{i}, \gamma_{i}(i=1,2,3)$ are free real parameters to be 
determined. Substituting Eq.(11) into Eq.(3) with computer algebra software Mathematic 12, we get two sets of parameter relations:

Case 1:

$$
\left\{\begin{array}{l}
a_{1}=\frac{a_{3} k_{3}}{k_{1}}, b_{1}=0, c_{1}=0, d_{1}=\frac{d_{3} k_{3}}{k_{1}}, \\
a_{2}=0, b_{2}=-\frac{l_{1} a_{3} c_{2}}{l_{2} d_{3}+l_{3} a_{3}^{3} k_{3}^{2}}, c_{2}=c_{2}, d_{2}=0, \\
a_{3}=a_{3}, b_{3}=0, c_{3}=0, d_{3}=d_{3} .
\end{array}\right.
$$

where $\delta_{i} k_{i} l_{i} \neq 0(i=1,2,3)$. A multi-breather solitary solution is constructed by inserting Eq.(11)and Eq.(12)into Eq.(3) as follows

$$
u(x, y, z, t)=\frac{6 l_{3}}{l_{4}}\left(\frac{a_{3}^{2} k_{3}^{2} \delta_{1} \cosh \phi_{1}+a_{3}^{2} k_{3}^{2} \delta_{3} \cosh \phi_{3}}{\delta_{1} \cosh \phi_{1}+\delta_{2} \cos \phi_{2}+\delta_{3} \cosh \phi_{3}}-\frac{\left(a_{3} k_{3} \delta_{1} \sinh \phi_{1}+a_{3} k_{3} \delta_{3} \sinh \phi_{3}\right)^{2}}{\left(\delta_{1} \cosh \phi_{1}+\delta_{2} \cos \phi_{2}+\delta_{3} \cosh \phi_{3}\right)^{2}}\right)
$$

where

$$
\left\{\begin{array}{l}
\phi_{1}=k_{1}\left(\frac{a_{3} k_{3}}{k_{1}} x+\frac{d_{3} k_{3}}{k_{1}} t+\gamma_{1}\right) \\
\phi_{2}=k_{2}\left(-\frac{l_{1} a_{3} c_{2}}{l_{2} d_{3}+l_{3} a_{3}^{3} k_{3}^{2}} y+c_{2} t+\gamma_{2}\right), \\
\phi_{3}=k_{3}\left(a_{3} x+d_{3} t+\gamma_{3}\right) .
\end{array}\right.
$$

The solution $u$ expressed in Eq.(13) is a kind of double breather wave solution, which takes on the characteristics of double solitary waves with the variable $\phi_{1}$ and $\phi_{3}$, and the characteristics of periodic solitary waves with the variable $\phi_{2}$. Besides, under the action of the function $\cos \phi_{2}, u$ also shows the characteristics of local oscillation. Therefore, the breather wave solutions with these characteristics are named double breathers $[38,39]$. The oscillation behaviour of double breathers are presented in Fig.4(a). In order to construct the rogue wave or lump-type solutions from Eq.(11), the parameters $\delta_{i}(i=1,2,3)$ must satisfy:

$$
\lim _{k_{i} \rightarrow 0}\left(\delta_{1}+\delta_{2}+\delta_{3}\right)=0
$$

so we take $k_{2}=r k_{1}, \delta_{1}=\cos \left(\tilde{i} m k_{1}\right), \delta_{2}=-2 \cosh \left(\tilde{i} n k_{1}\right), \delta_{3}=\cos \left(\tilde{i} s k_{1}\right)$, where $\tilde{i}$ is imaginary number, $m, n, r, s$ are real number. Letting $k_{1} \rightarrow 0$, lump solution are given by

$$
u(x, y, z, t)=\frac{6 l_{3}}{l_{4}}\left(\frac{4 a_{1}^{2}}{m^{2}+2 n^{2}+s^{2}+\theta_{1}^{2}+2 r^{2} \theta_{2}^{2}+\frac{a_{1}^{2}}{a_{3}^{2}} \theta_{3}^{2}}-\frac{4\left(a_{1} \theta_{1}+\frac{a_{1}^{2}}{a_{3}} \theta_{3}\right)^{2}}{\left(m^{2}+2 n^{2}+s^{2}+\theta_{1}^{2}+2 r^{2} \theta_{2}^{2}+\frac{a_{1}^{2}}{a_{3}^{2}} \theta_{3}^{2}\right)^{2}}\right)
$$

where

$$
\lim _{k_{i} \rightarrow 0} \frac{\phi_{i}}{k_{i}}=\theta_{i}(i=1,2,3) .
$$

$\theta_{1}=a_{1} x+\frac{a_{1} d_{3}}{a_{3}} t+\gamma_{1}, \theta_{2}=-\frac{l_{1} l_{3} a_{3} c_{2}}{l_{2} d_{3}} y+c_{2} z+\gamma_{2}, \theta_{3}=a_{3} x+d_{3} t+\gamma_{3}$. When $k_{1} \rightarrow 0$, the characteristics of period and breather of solution Eq.(13) are degenerated as a rational function solution in Eq.(16). Besides, the solution $u$ in Eq.(16) goes to 0 when $|x|,|y|,|z|,|t|$ tend to $\infty$ in any direction, and shows the characteristic of polynomial attenuation on all spatial variables. Meanwhile, we know that the solution is non-singular as the parameters satisfy $m^{2}+2 n^{2}+s^{2}>0$ from the expression of Eq.(16). From Fig.4(b), 
the lump solution $u$ has a upward peak and a downward valley, the lump solution with this structures is called rogue wave or lump-type solution $[15,17]$.

(a)

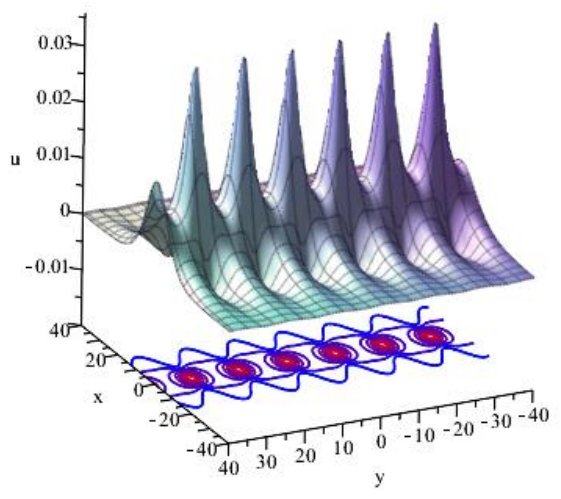

(b)

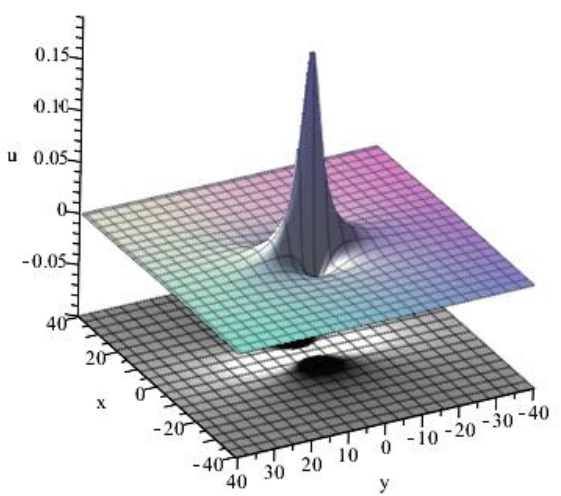

Fig. 4: (a)Spatial structure of double breather in Eq.(13) as $l_{1}=2, l_{2}=1, l_{3}=2, l_{4}=12, \delta_{1}=2, \delta_{2}=4, \delta_{3}=$ $5, k_{1}=\frac{1}{4}, k_{2}=10, k_{3}=\frac{1}{2}, a_{3}=\frac{1}{4}, c_{2}=1, d_{3}=10, \gamma_{i}=0(i=1,2,3), z=10, t=0$; (b)Spatial structure of lump wave in Eq.(16) as $l_{1}=1, l_{2}=1, l_{3}=1, l_{4}=12, a_{3}=d_{3}=r=1, c_{2}=-1, z=1, t=0$.

Case 2:

$$
\begin{aligned}
\delta_{3} & =0, a_{2}=0, b_{1}=\frac{a_{1} c_{1} l_{1}\left(\delta_{2}^{2}-\delta_{1}^{2}\right)}{\left(l_{2} d_{1}+4 l_{3} a_{1}^{3} k_{1}^{2}\right) \delta_{1}^{2}-\left(l_{2} d_{1}+l_{3} a_{1}^{3} k_{1}^{2}\right) \delta_{2}^{2}}, \\
d_{2} & =\frac{3 k_{1}^{4} l_{1} l_{3} a_{1}^{4} c_{1} \delta_{1}^{2}}{l_{2} k_{2}^{2} b_{2}\left(l_{2} d_{1}+4 l_{3} a_{1}^{3} k_{1}^{2} \delta_{1}^{2}-\left(l_{2} d_{1}+l_{3} a_{1}^{3} k_{1}^{2} \delta_{2}^{2}\right.\right.}, \\
c_{2} & =-\frac{b_{2}\left(l_{2} d_{1}+l_{3} k_{1}^{2} a_{1}^{3}\right)}{l_{1} a_{1}}-\frac{3 \delta_{1}^{2} k_{1}^{4} l_{1} l_{3} a_{1}^{4} c_{1}^{2}\left(\delta_{2}^{2}-\delta_{1}^{2}\right)}{k_{2}^{2} b_{2}\left(\left(l_{2} d_{1}+4 l_{3} a_{1}^{3} k_{1}^{2}\right) \delta_{1}^{2}-\left(l_{2} d_{1}+l_{3} a_{1}^{3} k_{1}^{2}\right) \delta_{2}^{2}\right)^{2}},
\end{aligned}
$$

so we get

$$
f(x, y, z, t)=\delta_{1} \cosh \left(k_{1}\left(a_{1} x+b_{1} y+c_{1} z+d_{1} t+\gamma_{1}\right)\right)+\delta_{2} \cos \left(k_{2}\left(b_{2} y+c_{2} z+d_{2} t+\gamma_{2}\right)\right)
$$

An exact one breather-wave solution of Eq.(1) is obtained by combining Eq.(17), (18) and (2), the spatial structure is shown in Fig.5(a).

Similarly, we take $k_{2}=r k_{1}, \delta_{1}=\cos \left(\imath l k_{1}\right), \delta_{2}=-\cosh \left(\imath s k_{1}\right), \imath$ is imaginary number, and let $k_{1} \rightarrow 0$. New type lump solution is obtained (Fig.5(b)).

$$
u(x, y, z, t)=\frac{6 l_{3}}{l_{4}}\left(\frac{2 a_{1}^{2}}{\zeta_{1}^{2}+r^{2} \zeta_{2}^{2}+l^{2}+s^{2}}-\frac{\left(2 a_{1} \zeta_{1}\right)^{2}}{\left(\zeta_{1}^{2}+r^{2} \zeta_{2}^{2}+l^{2}+s^{2}\right)^{2}}\right) .
$$

where $\zeta_{1}=a_{1} x-\frac{l_{1} a_{1} c_{1}\left(l^{2}+s^{2}\right)}{3 l_{3} a_{1}^{3}+l_{2} d_{1}\left(l^{2}+s^{2}\right)} y+c_{1} z+d_{1} t+\gamma_{1}, \zeta_{2}=b_{2} y-\left(\frac{l_{2} b_{2} d_{1}}{l_{1} a_{1}}+\frac{3 l_{1} l_{3} a_{1}^{4} c_{1}^{2}\left(l^{2}+s^{2}\right)}{r^{2} b_{2}\left(3 l_{3} a_{1}^{3}+l_{2} d_{1}\left(l^{2}+s^{2}\right)\right)^{2}}\right) z+$ $+\frac{3 l_{1} l_{3} a_{1}^{4} c_{1}}{r^{2} l_{2} b_{2}\left(3 l_{3} a_{1}^{3}+l_{2} d_{1}\left(l^{2}+s^{2}\right)\right)} t+\gamma_{2}$ and $l^{2}+s^{2}>0$. 


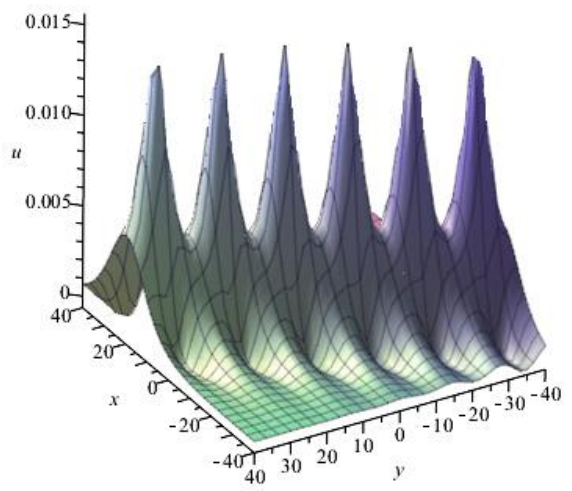

(b)

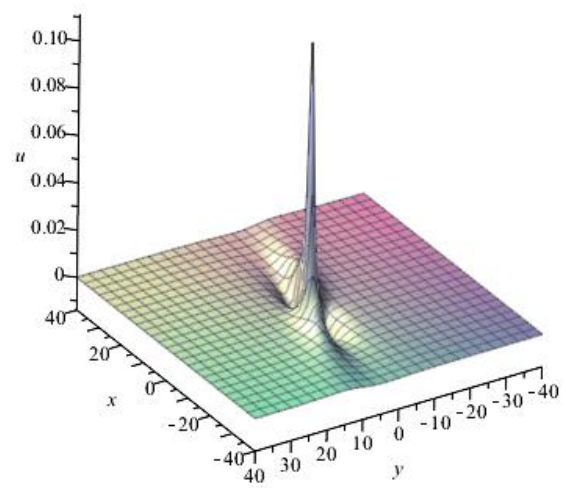

Fig. 5: (a)Spatial structure of breather in Eq.(18) as $l_{1}=1, l_{2}=1, l_{3}=1, l_{4}=12, \delta_{1}=\frac{1}{5}, \delta_{2}=\frac{1}{10}, k_{1}=1, k_{2}=$ $2, a_{1}=\frac{1}{8}, b_{2}=4, c_{1}=\frac{1}{2}, c_{2}=1, d_{1}=10, \gamma_{i}=0(i=1,2,3), z=1, t=0$; (b)Spatial structure of lump wave in Eq.(19) as $l_{1}=1, l_{2}=1, l_{3}=1, l_{4}=12, a_{1}=r=1, c_{2}=-1, d_{1}=\frac{1}{6}, l=s=\frac{3 \sqrt{2}}{2}, z=1, t=0$.

\subsection{Full degeneration of $N$-solitons to $M$-lump solutions}

In this subsection, we study $M$-lump solutions of Eq.(1) by long wave limit method. In order to obtain $M$-lump $(N=2 M)$ solutions, we need take each $\exp \left(\eta_{i}^{(0)}\right)=-1, k_{i} / k_{j}=O(1), p_{i}=O(1), q_{i}=O(1)$ and $k_{i} \rightarrow 0$ in Eq.(4) and (5). Then we get

$$
f=f_{N}=\sum_{\mu=0,1} \prod_{i=1}^{N}(-1)^{\mu_{i}}\left(1+\mu_{i} k_{i} \theta_{i}\right) \prod_{i<j}^{(N)}\left(1+\mu_{i} \mu_{j} k_{i} k_{j} B_{i j}\right)+O\left(k^{N+1}\right) .
$$

For the sake of simplification, we omit constant factor $\prod_{i=1}^{N} k_{i}$ in $f_{N}$ and get the simplified form as follows:

$$
\begin{aligned}
f_{l}^{[M]} & =\prod_{i=1}^{N} \theta_{i}+\frac{1}{2} \sum_{i, j}^{N} B_{i j} \prod_{l \neq i, j}^{N} \theta_{l}+\frac{1}{2 ! 2^{2}} \sum_{i, j, r, s}^{N} B_{i j} B_{s r} \prod_{l \neq i, j, s, r}^{N} \theta_{l}+\cdots \\
& +\frac{1}{M ! 2^{M}} \sum_{i, j, \cdots, m, n}^{N} \overbrace{B_{i j} B_{k l} \cdots B_{m n}}^{N} \prod_{p \neq i, j, k, l, \cdots, m, n}^{N} \theta_{p}+\cdots
\end{aligned}
$$

where

$$
\theta_{i}=x+p_{i} y+q_{i} z-\frac{l_{1} q_{i}}{l_{2} p_{i}} t, B_{i j}=\frac{6 l_{3} p_{i} p_{j}\left(p_{i}+p_{j}\right)}{l_{1}\left(p_{i}-p_{j}\right)\left(p_{i} q_{j}-p_{j} q_{i}\right)} .
$$

If we take $p_{M+i}=p_{i}^{*}, q_{M+i}=q_{i}^{*}$ with the condition $B_{i j}>0$, a class of $M$-lump solutions can be constructed.

When $N=2$, the expression $f$ in Eq.(21) is written as

$$
f_{2}=\theta_{1} \theta_{2}+B_{12}
$$




$$
B_{12}=\frac{6 l_{3} p_{1} p_{2}\left(p_{1}+p_{2}\right)}{l_{1}\left(p_{1}-p_{2}\right)\left(p_{1} q_{2}-p_{2} q_{1}\right)} .
$$

Taking $p_{1}=p_{2}^{*}=a_{1}+b_{1} i, q_{1}=q_{2}^{*}=c_{1}+d_{1} i$, the 1-lump $u_{l}^{[1]}=\frac{6 l_{3}}{l_{4}}\left(\ln f_{l}^{[1]}\right)_{x x}$ with the $f_{l}^{[1]}$ as (see Fig.6(a))

$$
f_{l}^{[1]}=\left(x^{\prime}+a_{1} y^{\prime}+c_{1} z^{\prime}-\frac{l_{1}\left(a_{1} c_{1}+b_{1} d_{1}\right)}{l_{2}\left(a_{1}^{2}+b_{1}^{2}\right)} t^{\prime}\right)^{2}+\left(b_{1} y^{\prime}+d_{1} z^{\prime}-\frac{l_{1}\left(a_{1} d_{1}-b_{1} c_{1}\right)}{l_{2}\left(a_{1}^{2}+b_{1}^{2}\right)} t^{\prime}\right)^{2}+\Gamma_{3} .
$$

where

$$
\begin{aligned}
& x^{\prime}=x-\frac{l_{1}\left(a_{1} c_{1}+b_{1} d_{1}\right)}{l_{2}\left(a_{1}^{2}+b_{1}^{2}\right)} t, \\
& y^{\prime}=y+\frac{l_{1} c_{1}}{l_{2}\left(a_{1}^{2}+b_{1}^{2}\right)} t, \\
& z^{\prime}=z-\frac{l_{1} a_{1}}{l_{2}\left(a_{1}^{2}+b_{1}^{2}\right)} t, \\
& \Gamma_{3}=-\frac{l_{1}\left(a_{1} c_{1}+b_{1} d_{1}\right)}{l_{2}\left(a_{1}^{2}+b_{1}^{2}\right)}>0 .
\end{aligned}
$$

When $a_{1} \neq 0$, the trajectory can be defined along the path $(x(t), y(t))$ as follows

$$
\left\{\begin{array}{l}
x+a_{1} y+c_{1} z+\Gamma_{1} t= \pm \sqrt{\Gamma_{3}}, \\
b_{1} y+d_{1} z+\Gamma_{2} t=0 .
\end{array}\right.
$$

with

$$
\begin{aligned}
& \Gamma_{1}=-\frac{l_{1}\left(a_{1} c_{1}+b_{1} d_{1}\right)}{l_{2}\left(a_{1}^{2}+b_{1}^{2}\right)}, \\
& \Gamma_{2}=-\frac{l_{1}\left(a_{1} d_{1}-b_{1} c_{1}\right)}{l_{2}\left(a_{1}^{2}+b_{1}^{2}\right)} .
\end{aligned}
$$

On $(x, y)$-plane, when $z=0$ the lump reaches the $u_{\max }=\frac{4 b_{1}\left(a_{1} d_{1}-b_{1} c_{1}\right)}{3 l_{3} a_{1}\left(a_{1}^{2}+b_{1}^{2}\right)}$ at the point $\left(-\frac{l_{1}\left(a_{1}^{2} d_{1}-2 a_{1} b_{1} c_{1}+b_{1}^{2} d_{1}\right) t}{l_{2} b_{1}\left(a_{1}^{2}+b_{1}^{2}\right)}, \frac{l_{1}\left(a_{1} d_{1}-b_{1} c_{1}\right) t}{l_{2} b_{1}\left(a_{1}^{2}+b_{1}^{2}\right)}\right)$, and reaches the $u_{\text {min }}=\frac{4 l_{1} b_{1}\left(a_{1} d_{1}-b_{1} c_{1}\right)\left(1+3 a_{1} b_{1}^{2} l_{3}+3 a_{1}^{3} l_{3}\right)}{3 l_{3} a_{1}\left(a_{1}^{2}+b_{1}^{2}\right)\left(-1+3 a_{1} b_{1}^{2} l_{3}+3 a_{1}^{3} l_{3}\right)}$ at $\left(-\frac{l_{1}\left(a_{1}^{2} d_{1}-2 a_{1} b_{1} c_{1}+b_{1}^{2} d_{1}\right) t}{l_{2} b_{1}\left(a_{1}^{2}+b_{1}^{2}\right)}-\frac{3 l_{3} a_{1}\left(a_{1}^{2}+b_{1}^{2}\right)}{\sqrt{l_{1} b_{1}\left(b_{1} c_{1}-a_{1} d_{1}\right)}}, \frac{l_{1}\left(a_{1} d_{1}-b_{1} c_{1}\right) t}{l_{2} b_{1}\left(a_{1}^{2}+b_{1}^{2}\right)}\right)$ and $\left(-\frac{l_{1}\left(a_{1}^{2} d_{1}-2 a_{1} b_{1} c_{1}+b_{1}^{2} d_{1}\right) t}{l_{2} b_{1}\left(a_{1}^{2}+b_{1}^{2}\right)}+\frac{3 l_{3} a_{1}\left(a_{1}^{2}+b_{1}^{2}\right)}{\sqrt{l_{1} b_{1}\left(b_{1} c_{1}-a_{1} d_{1}\right)}}, \frac{l_{1}\left(a_{1} d_{1}-b_{1} c_{1}\right) t}{l_{2} b_{1}\left(a_{1}^{2}+b_{1}^{2}\right)}\right)$.

When $N=4$, Eq.(21) can be reduced to the following expression:

$f_{l}^{[2]}=\theta_{1} \theta_{2} \theta_{3} \theta_{4}+B_{12} \theta_{3} \theta_{4}+B_{13} \theta_{2} \theta_{4}+B_{14} \theta_{2} \theta_{3}+B_{23} \theta_{1} \theta_{4}+B_{24} \theta_{1} \theta_{3}+B_{34} \theta_{1} \theta_{2}+B_{12} B_{34}+B_{13} B_{24}+B_{14} B_{23}$,

where

$$
\theta_{i}=x+p_{i} y+q_{i} z-\frac{l_{1} q_{i}}{l_{2} p_{i}} t, B_{i j}=\frac{6 l_{3} p_{i} p_{j}\left(p_{i}+p_{j}\right)}{l_{1}\left(p_{i}-p_{j}\right)\left(p_{i} q_{j}-p_{j} q_{i}\right)} .
$$

2-lump (Fig.6(b)) will be obtained by taking $p_{1}=p_{3}^{*}=a_{1}+b_{1} i, p_{2}=p_{4}^{*}=a_{2}+b_{2} i, q_{1}=q_{3}^{*}=c_{1}+d_{1} i$, $q_{2}=q_{4}^{*}=c_{2}+d_{2} i$.

When $N=6$, taking $p_{1}=p_{4}^{*}=a_{1}+b_{1} i, p_{2}=p_{5}^{*}=a_{2}+b_{2} i, p_{3}=p_{6}^{*}=a_{3}+b_{3} i, q_{1}=q_{4}^{*}=c_{1}+d_{1} i$, $q_{2}=q_{5}^{*}=c_{2}+d_{2} i, q_{3}=q_{6}^{*}=c_{3}+d_{3} i$ into Eq.(21), we obtain 3-lump solution (Fig.6(c)).

Therefore, we find that a $M$-lump can be degenerated from a $N$-order soliton. From Fig.6, the lump waves include both bright waves and dark waves, and the shapes will change obviously with energy transformation between them as no elastic interaction occurs, and the different parameter values are 


$$
\begin{aligned}
& l_{1}=2, l_{2}=1, l_{3}=1, l_{4}=3, z=0, t=0, \text { (a) } p_{1}=-\frac{1}{2}+i, p_{2}=-\frac{1}{2}-i, q_{1}=-1+3 i, q_{2}=-1-3 i . \text { (b) } \\
& p_{1}=-\frac{1}{2}+i, p_{2}=-\frac{1}{10}-i, p_{3}=-\frac{1}{2}-i, p_{4}=-\frac{1}{10}+i, q_{1}=-1+3 i, q_{2}=-5-8 i, q_{3}=-1-3 i, q_{4}=-5+8 i . \\
& \text { (c) } p_{1}=-\frac{1}{2}+i, p_{2}=-\frac{1}{10}-i, p_{3}=\frac{1}{6}+\frac{1}{6} i, p_{4}=-\frac{1}{2}+i, p_{5}=-\frac{1}{10}+i, p_{6}=\frac{1}{6}-\frac{1}{6} i, q_{1}=-1+3 i, q_{2}= \\
& -5-8 i, q_{3}=\frac{1}{3}+\frac{1}{2} i, q_{4}=-1-3 i, q_{5}=-5+8 i, q_{6}=\frac{1}{3}-\frac{1}{2} i .
\end{aligned}
$$
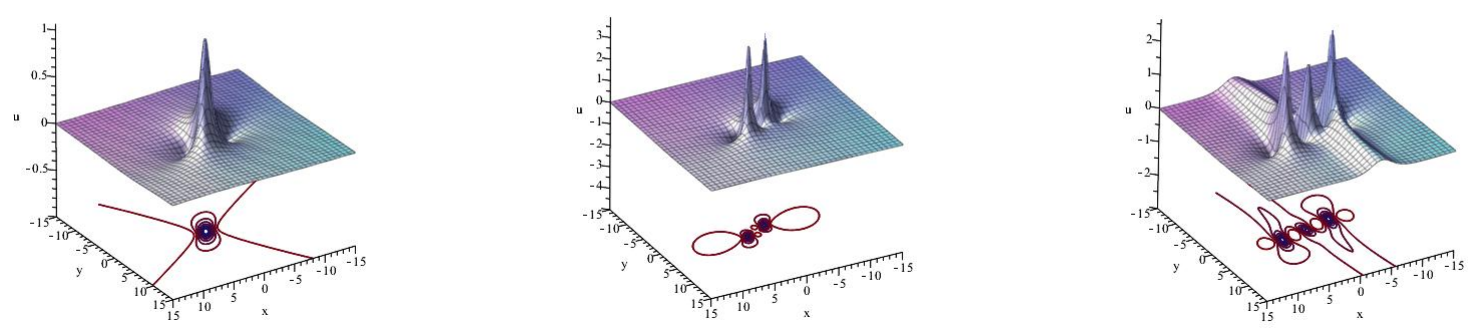

Fig. 6: Spatial evolution behavior of $M$-lump $u_{l}^{[M]}$ with the increasing number (a) $M=1$, (b) $M=2$, (c) $M=3$.

\section{The hybrid solutions}

In this section, abundant hybrid solutions and dynamics behaviours can be obtained during partial degeneration of $\mathrm{N}$-soliton. Several kinds of interaction solutions consisting of breathers and solitons, lump waves and solitons are discussed based on above sections.

\section{$4.1(2 H+K)$-order hybrid solutions}

Interaction solutions composed of breathers and solitons, we take the parameters in Eq.(4) as

$$
\begin{gathered}
N=2 H+K, k_{j}=k_{H+j}^{*}=k_{j 1}+i k_{j 2}, p_{j}=p_{H+j}^{*}=p_{j 1}+i p_{j 2}, j=1,2, \cdots, H \\
k_{t}=k_{t}, p_{t}=p_{t}, t=2 H+1,2 H+2, \cdots, 2 H+K, q_{i}=q_{i}, \eta_{i}^{(0)}=0, i=1,2, \cdots, 2 H+K .
\end{gathered}
$$

$k_{t}, p_{t}$ and $q_{i}$ are real numbers. Interaction solutions for Eq.(1) between $H$-breathers and $K$-soliton are constructed.

When $N=3$, interaction solutions between 1-breather and 1-soliton are constructed with

$$
k_{1}=k_{2}^{*}=k_{11}+i k_{12}, k_{3}=k_{3}, p_{1}=p_{2}^{*}=p_{11}+i p_{12}, p_{3}=p_{3}, q_{i}=q_{i}, i=1,2,3 .
$$

As it is shown in Fig.7(a), the 1-breather and 1-soliton are intersected and keep colliding with each other on the $(x, y)$-plane with $l_{1}=2, l_{2}=1, l_{3}=1, l_{4}=3, z=0, t=0, k_{1}=\frac{1}{2}+i, k_{2}=\frac{1}{2}-i, k_{3}=1, p_{1}=$ $1+\frac{1}{2} i, p_{2}=1-\frac{1}{2} i, p_{3}=1, q_{1}=q_{2}=q_{3}=-1$.

When $N=4$, interaction solutions between 1-breather and 2-soliton are constructed with

$$
k_{1}=k_{2}^{*}=k_{11}+i k_{12}, k_{3}=k_{3}, k_{4}=k_{4}, p_{1}=p_{2}^{*}=p_{11}+i p_{12}, p_{3}=p_{3}, p_{4}=p_{4}, q_{i}=q_{i}, i=1,2,3,4 .
$$


As it is shown in Fig.8(a), the 1-breather and 2-soliton are interacting with each other on the $(x, y)$-plane with $l_{1}=2, l_{2}=1, l_{3}=1, l_{4}=3, z=0, t=0, k_{1}=\frac{1}{2}+i, k_{2}=\frac{1}{2}-i, k_{3}=1, k_{4}=1, p_{1}=1+\frac{1}{2} i, p_{2}=$ $1-\frac{1}{2} i, p_{3}=2, p_{4}=\frac{3}{4}, q_{1}=q_{2}=q_{3}=q_{4}=-1$.

Similarly, taking $N=5$ :

Case I. The 1-breather and 3-soliton are interacting with each other on the $(x, y)$-plane (Fig.9(a)) with

$$
\begin{gathered}
k_{1}=k_{2}^{*}=k_{11}+i k_{12}, k_{3}=k_{3}, k_{4}=k_{4}, k_{5}=k_{5}, p_{1}=p_{2}^{*}=p_{11}+i p_{12}, \\
p_{3}=p_{3}, p_{4}=p_{4}, p_{5}=p_{5}, q_{i}=q_{i}, i=1,2,3,4,5
\end{gathered}
$$

specific parameters taking

$$
\begin{gathered}
l_{1}=2, l_{2}=1, l_{3}=1, l_{4}=3, z=0, t=0, k_{1}=\frac{1}{2}+i, k_{2}=\frac{1}{2}-i, k_{3}=k_{4}=k_{5}=1, \\
p_{1}=1+\frac{1}{2} i, p_{2}=1-\frac{1}{2} i, p_{3}=2, p_{4}=\frac{3}{4}, p_{4}=\frac{1}{4}, q_{1}=q_{2}=q_{3}=q_{4}=q_{5}=-1 .
\end{gathered}
$$

Case II. The interaction between 2-breather and 1-soliton on the $(x, y)$-plane is shown in Fig.10(a) with

$$
\begin{gathered}
k_{1}=k_{2}^{*}=k_{11}+i k_{12}, k_{3}=k_{4}^{*}=k_{21}+i k_{22}, k_{5}=k_{5}, p_{1}=p_{2}^{*}=p_{11}+i p_{12}, \\
p_{3}=p_{4}^{*}=p_{21}+i p_{22}, p_{5}=p_{5}, q_{1}=q_{2}^{*}=q_{11}+i q_{12}, q_{3}=q_{4}^{*}=q_{21}+i q_{22}, q_{5}=q_{5} .
\end{gathered}
$$

and

$l_{1}=2, l_{2}=1, l_{3}=1, l_{4}=3, z=0, t=0, k_{1}=\frac{1}{2}+i, k_{2}=\frac{1}{2}-i, k_{3}=\frac{1}{2}+i, k_{4}=\frac{1}{2}-i, k_{5}=1, p_{1}=1+\frac{1}{2} i$,

$p_{2}=1-\frac{1}{2} i, p_{3}=1+i, p_{4}=1-i, p_{5}=-\frac{1}{2}, q_{1}=-\frac{1}{2}+i, q_{2}=-\frac{1}{2}-i, q_{3}=-\frac{1}{2}+i, q_{4}=-\frac{1}{2}-i, q_{5}=-\frac{1}{2}$.

According to the characteristic of the figures and propagating mechanism, above interactions are elastic.

\section{$4.2(2 H+L)$-order hybrid solutions}

Interaction solutions composed of $H$-lump and $L$-solitons for Eq.(1) by taking the parameters in Eq.(4) as follows:

$$
N=2 H+L, k_{i}=k_{i}, e^{\eta_{i}^{(0)}}=-1, k_{i} \rightarrow 0, i=1,2, \cdots, 2 H .
$$

Firstly, setting $N=3, H=1, L=1$, elastic interaction solution between 1-lump and 1-soliton is displayed in Fig.7(b) with

$$
f_{3}=\theta_{1} \theta_{2}+B_{12}+\left(\theta_{1} \theta_{2}+B_{12}+B_{23} \theta_{1}+B_{13} \theta_{2}+B_{13} B_{23}\right) e^{\eta_{3}}
$$

and

$$
l_{1}=2, l_{2}=1, l_{3}=1, l_{4}=3, z=0, t=0, k_{1}=\frac{1}{2}+i, k_{2}=\frac{1}{2}-i, k_{3}=1,
$$




$$
p_{1}=1+\frac{1}{2} i, p_{2}=1-\frac{1}{2} i, p_{3}=\frac{1}{20}, q_{1}=q_{2}=q_{3}=-3 .
$$

Secondly, letting $N=4, H=1, L=2$, elastic interaction solution between 1-lump and 2-soliton is displayed in Fig.8(b) with

$$
\begin{aligned}
f_{4}=\theta_{1} \theta_{2}+B_{12}+\left(\theta_{1} \theta_{2}\right. & \left.+B_{12}+B_{23} \theta_{1}+B_{13} \theta_{2}+B_{13} B_{23}\right) e^{\eta_{3}}+\left(\theta_{1} \theta_{2}+B_{12}+B_{24} \theta_{1}+B_{14} \theta_{2}+B_{14} B_{24}\right) e^{\eta_{4}} \\
& +\left(B_{12}+\left(B_{13}+B_{14}+\theta_{1}\right)\left(B_{23}+B_{24}+\theta_{2}\right)\right) e^{\eta_{3}+\eta_{4}+A_{34}} .
\end{aligned}
$$

and

$$
\begin{gathered}
l_{1}=2, l_{2}=1, l_{3}=1, l_{4}=3, z=0, t=0, k_{1}=\frac{1}{2}+i, k_{2}=\frac{1}{2}-i, k_{3}=k_{4}=1, \\
p_{1}=1+\frac{1}{2} i, p_{2}=1-\frac{1}{2} i, p_{3}=\frac{1}{20}, p_{4}=1, q_{1}=q_{2}=-3, q_{3}=q_{4}=-1 .
\end{gathered}
$$

Fig.8(c) depicts the elastic collision between 1-lump and 1-breather with

$$
\begin{aligned}
& l_{1}=2, l_{2}=1, l_{3}=1, l_{4}=3, z=0, t=0, k_{1}=i, k_{2}=-i, k_{3}=i, k_{4}=-i, \\
& p_{1}=\frac{1}{2}+2 i, p_{2}=\frac{1}{2}-2 i, p_{3}=1+i, p_{4}=1-i, q_{1}=q_{2}=q_{3}=q_{4}=-3 .
\end{aligned}
$$

When $N=5, H=1, L=3$, with $l_{1}=2, l_{2}=1, l_{3}=1, l_{4}=3, z=0, t=0, k_{1}=\frac{1}{2}+i, k_{2}=\frac{1}{2}-i, k_{3}=$ $k_{4}=k_{5}=1, p_{1}=1+\frac{1}{2} i, p_{2}=1-\frac{1}{2} i, p_{3}=3, p_{4}=\frac{3}{4}, p_{5}=\frac{1}{8}, q_{1}=q_{2}=-1, q_{3}=3, q_{4}=q_{5}=-1$, elastic interaction solution between 1-lump and 3-soliton is displayed in Fig.9(b).

Finally, elastic interaction solution between 2-lump and 1-soliton is displayed in Fig.10(b) with $l_{1}=$ $2, l_{2}=1, l_{3}=1, l_{4}=3, z=0, t=0, p_{1}=\frac{1}{2}+i, p_{2}=-\frac{1}{10}-i, p_{3}=\frac{1}{2}-i, p_{4}=-\frac{1}{10}+i, p_{5}=-\frac{1}{2}, q_{1}=$ $-1+3 i, q_{2}=-5-8 i, q_{3}=-1-3 i, q_{4}=-5+8 i, q_{5}=-\frac{1}{2}$.
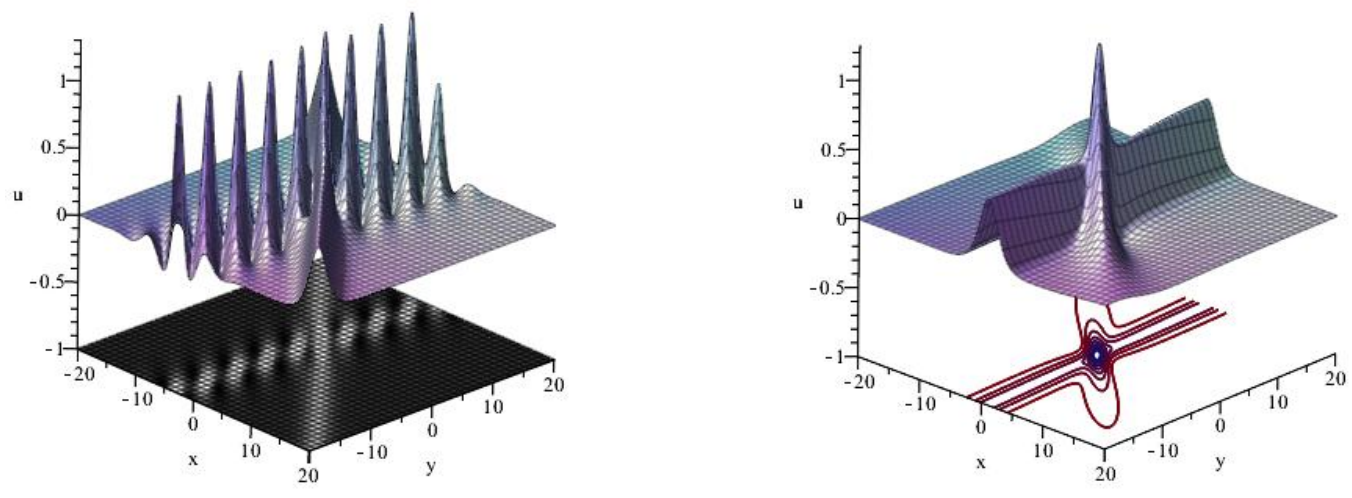

Fig. 7: (a) Interaction phenomenon between 1-breather and 1-soliton, (b)Collision between 1-lump and 1-soliton. 

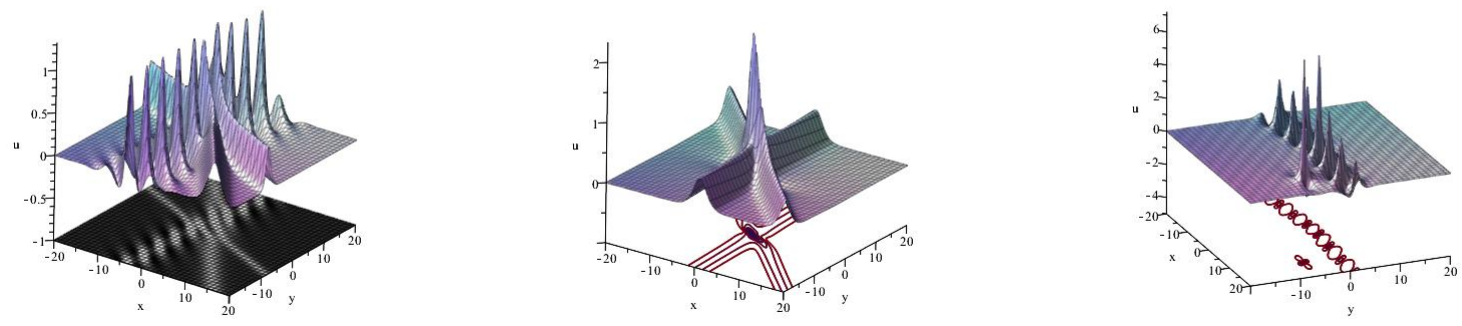

Fig. 8: Different interaction phenomenon including (a) 1-breather and 2-soliton, (b) 1-lump and 2-soliton, (c) 1-lump and 1-breather.

(a)

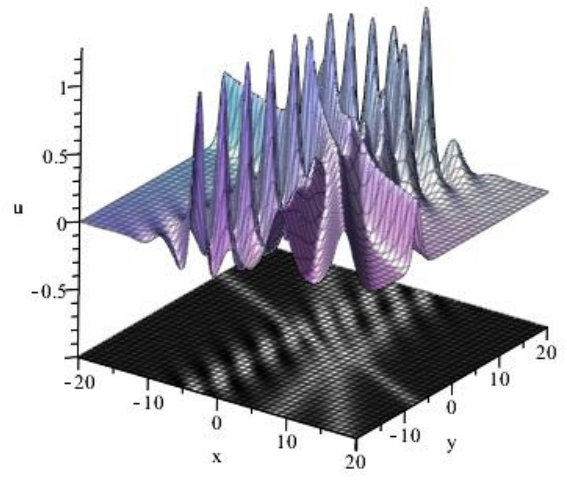

(b)

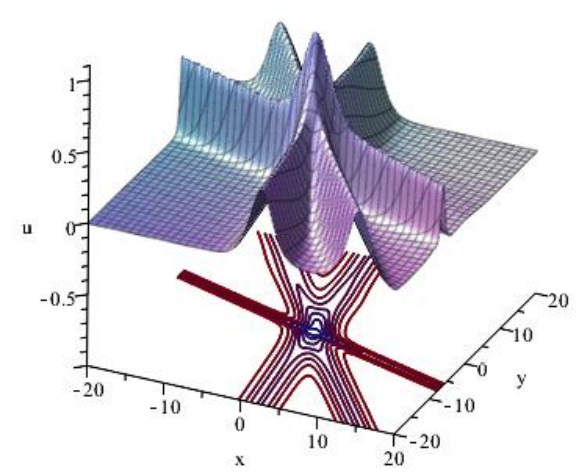

Fig. 9: Two kinds of interaction phenomenon including (a) 1-breather and 3-soliton, (b)1-lump and 3-soliton.

\section{Conclusion}

As the high-dimensional NLEEs can describe many real-world phenomenon more accurately, so the study of high-dimensional NLEEs has attracted great interest of mathematical physics researchers. In this manuscript, $N$-soliton solutions are constructed based on symbolic computation and Hirota bilinear form. In the process of degeneration of $N$-soliton solutions, $T$-breathers $(T=1,2,3, \cdots)$ are derived by taking complexication method, Fig.1,2,3 depict the specific shape and dynamic behaviors, and the collision of breathers is elastic. Then rogue waves expressed in Eq.(16) and (19) are found out during the degeneration of breathers by taking the parameter limit method, Fig. 4 and 5 show the process of the evolution. Through full degeneration of $N$-soliton, $M$-lump $\left(M=\frac{N}{2}=1,2,3, \cdots\right)$ solutions are derived 


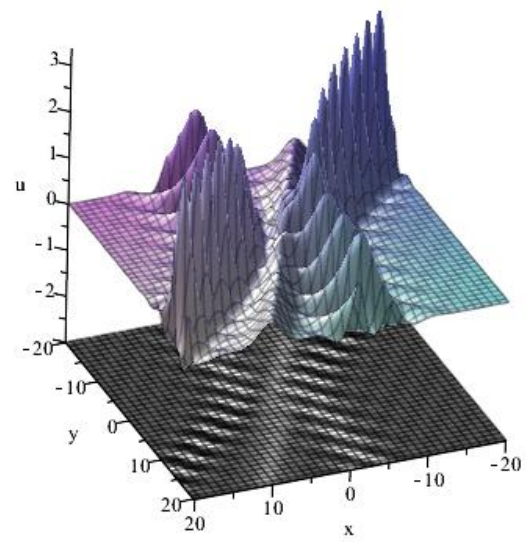

(b)

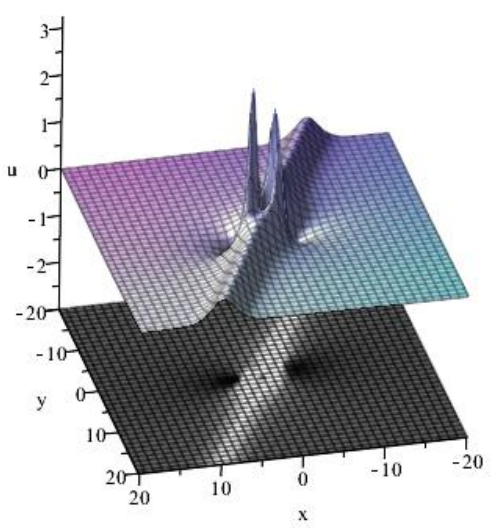

Fig. 10: Interaction solutions composed of (a) 2-breather and 1-soliton, (b) 2-lump and 1-soliton.

based on long wave limit approach as shown in Fig.6. If the $N$-soliton degenerates partially, the abundant hybrid solutions composed of soliton, breather and lump can be constructed, such as interaction between breather and soliton shown in the first plot of Fig.7,8,9,10, interaction between lump and soliton shown in the second plot of Fig.7,8,9,10, interaction between lump and breather shown in Fig.8(c).The dynamic properties of the solutions have been analyzed by taking appropriate parameters. This work greatly enrich the dynamic behavior of Eq.(1), and the methods used to obtain new exact solutions also can be extended to solve other nonlinear partial different equations. For some important classical mathematical and physical models, the methods of studying solutions are very significant.

\section{Acknowledgments}

The authors would like to express their sincere thanks to referees for their enthusiastic guidance and help. This work was supported by National Natural Science Foundation of China, Grant No.11901345, and Scientific and Technological Innovation Team of Nonlinear Analysis and Algebra with Their Applications in Universities of Yunnan Province, China, Grant No.2020CXTD25.

\section{Declarations}

Conflict of interest The authors declare that there are no conflicts of interests with publication of this work.

Ethical standards The authors ensure the compliance with ethical standards for this work. 


\section{Data availability statement}

The authors declare that data supporting the findings of this study are available within the article, the figures are concrete expression.

\section{References}

[1] Ablowitz, M.J.: Nonlinear dispersive waves: asymptotic analysis and solitons. Combridge: Combridge Univ. Press (2011)

[2] Vreugdenhil, C.B.: Numerical methods for shallow-water flow. London: Springer (2013)

[3] Ivanov, S.K., Kamchatnov, A.M.: Evolution of wave pulses in fully nonlinear shallow-water theory. Phys. Fluids. 31, $057102(2019)$

[4] Zabusky, N.J., Kruskal, M.D.: Interaction of solitons in a collisionless plasma and the recurrence of initial states. Phys. Rev. Lett. 15(6), 240-243 (1965)

[5] Kivshar, Y.S., Malomed, B.A.: Dynamics of solitons in nearly integrable system. Rev. Mod. Phys. 61(4), 763-915 (1989)

[6] Mihalache, D.: Mulitidimensional localized structures in optics and Bose-Einstein condensates: a selection of recent studies. J. Roman. Phys. 59(3), 295-312 (2014)

[7] Forte, S.: Quantum mechanics and field theory with fractional spin and statistics. Appl. Phys. Lett. 64(1), 193-236 (1992)

[8] Kibler, B., Fatome, J., Finot, C., Millot, G,, Dias, F., Genty, G., Akhmediev, N., Dudley, J.M.: The peregrine soliton in nonlinear fibre optics. Nat. Phys. 6(10), 790-795 (2010)

[9] Malomed, B., Torner, L., Wise, F., Mihalache, D.: On multidimensional solitons and their legacy in contemporary atomic, molecular and optical physics. J. Phys. B. At. Mol. Opt. Phys. 49(17), 170502 (2016)

[10] Dai, Z.D., Wang, C.J., Liu, J.: Inclined periodic homoclinic breather and rogue waves for the (1+1)dimensional Boussinesq equation. Pramana. J. Phys. 83(4), 473-480 (2014)

[11] Wang, C.J., Dai, Z.D., Lin, L.: Exact three-wave solution for higher dimensional KdV-type equation. Appl. Math. Comput. 216(2), 501-505 (2010)

[12] Ma, W.X.: Lump solutions to the Kadomtsev-Petviashvili equation. Phys. Lett. A 379, 1975-1978 (2015)

[13] Ma, W.X., Yong, X.L., Zhang, H.Q.: Diversity of interaction solutions to the (2+1)-dimensional Ito equation. Comput. Math. Appl. 75(1), 289-295 (2018)

[14] Ma, C.H., Deng, A.P.: Lump solution of (2+1)-dimensional Boussineaq equation. Commun. Theor. Phys. 65(5), 546-552 (2016) 
[15] Ma, W.X., and Zhou, Y.: Lump solutions to nonlinear partial different equations via Horita bilinear forms. J. Diff. Equ. 264(4), 2633-2659 (2018)

[16] Zhao, Z.L., Chen, Y., Han, B.: Lump soliton, mixed lump stripe and periodic lump solutions of a (2+1)dimensional asymmetrical Nizhnik-Novikov-Veselov equation. Mod. Phys. Lett. B 31(14), 1750157 (2017)

[17] Wang, C.J.: Spatiotemporal deformation of lump solution to (2+1)-dimensional KdV equation. Nonlinear Dynam. 84(2), 697-702 (2016)

[18] Peng, W.Q., Tian, S.F., Zhang, T.T.: Analysis on lump, lump off and rogue waves with predictability to the (2+1)-dimensional B-type Kadomtsev-Petviashvili equation. Phys. Lett. A 382, 2701-2708 (2018)

[19] Manakov, M.Q., Zakharov, V.E., Bordag, L.A.: Analysis on lump, Two-dimensional solitons of the KadomtsevPetviashvili equation and their interaction. Phys. Lett. A 63(3), 205-206 (1977)

[20] Ablowitz, M.J., Satsuma, J.: Solitons and rational solutions of nonlinear evolution equations. J. Math. Phys. 19(10), 2180-2186 (1978)

[21] Satsum, J., Ablowitz, M.J.: Two-dimensional lumps in nonlinear dispersive system. J. Math. Phys. 20(7), 1496-1503 (1979)

[22] Zhao, Z.L., He, L.C.: $M$-lump and hybrid solutions of a generalized (2+1)-dimensional Hirota-Satsuma-Ito equation. Appl. Math. Lett. 111, 106612 (2021)

[23] Guo, H.D., Xia, T.C., Hu, B.B.: Dynamics of abundant solutions to the $(3+1)$-dimensional generalized Yu-Toda-Sasa-Fukuyama equation. Appl. Math. Lett. 105, 106301 (2020)

[24] Tan, W., Dai, Z.D., Xie, J.L., Yin, Z.Y.: Dynamics of multi-breathers, $N$-solitons and $M$-lump solutions in the (2+1)-dimensional KdV equation. Nonlinear Dyn. 96, 1605-1614 (2019)

[25] Ma, W.X., Zhou,Y., Dougherty, R.: Lump-type solutions to nonlinear differential equations derived from generalized bilinear equations. Int. J. Mod. Phys. B 30, 1640018 (2016)

[26] Zhang, R.F., Li, M.C., Albishari, M., Zheng, F.C., Lan, Z.Z.: Generalized lump solutions, classical lump solutions and rogue waves of the (2+1)-dimensional Caudrey-Dodd-Gibbon-Kotera-Sawada-like equation. App. Math. Compu. 403, 126201 (2021)

[27] Ren, B., Lin, J., Lou, Z.M.: Lump and their interaction solutions of a $(2+1)$-dimensional generalized potential Kadomtsev-Petviashvili equation. J. App. Anal. Comput. 10(3) 935-945 (2020)

[28] Guo, Y.F., Dai, Z.D., Guo, C.X.: Lump solutions and interaction solutions for (2+1)-dimensional KPI equation. Front. Math. China 9, 1-12 (2021) http://doi.org/10.1007/s11464-021-0973-y

[29] Wang, H., Tian, S.F., Chen, Y., Zhang, T.T.: Dynamics of kink solitary waves and lump waves with interaction phenomena in a generalized (3+1)-dimensional Kadomtsev-Petviashvili-Boussinesq equation. Int. J. Comput. Math. 97(11), 2178-2190 (2020)

[30] Verma, P., and Kaur, L.: Integrability, bilinearization and analytic study of new form of (3+1)-dimensional B-type Kadomtsev-Petviashvili(BPK)-Boussinesq equation. Appl. Math. Comput. 346, 879-886 (2019) 
[31] Wang, C.J., Fang, H., Tang, X.X.: State transition of lump-type waves for the (2+1)-dimensional generalized KdV equation. Nonlinear Dyn. 95, 2943-2961 (2019)

[32] Tan, W.: Some new dynamical behaviour of double breathers and lump- $N$-solitons for the Ito equation. Int. J. Comput. Math. 98(5), 961-974 (2021)

[33] Tan, W., Dai, Z.D.: Dynamics of kinky wave for (3+1)-dimensional potential Yu-Tada-Sasa-Fukuyama equation. Nonlinear Dyn. 85(2), 817-823 (2016)

[34] Tan, W., Dai, Z.D., Xie, J.L., Qiu, D.Q.: Parameter limit method and its application in the (4+1)dimensional Fokas equation. Compu. Math. App. 75(12), 4214-4220 (2018)

[35] Yuan, F., Cheng, Y., He, J.S.: Degeneration of breathers in the Kadomtsev-Petviashvili I equation. Nonlinear Sci. Numer. Simul. 83, 105027 (2019)

[36] Guo, H.D., Xia, T.C., Hu, B.B.: High-order lumps, high-order breathers and hybrid solutions for an extend (3+1)-dimensional Jimbo-Miwa equation in fluid dynamics. Nonlinear Dyn. 100(1), 601-614 (2020)

[37] Zhang, R.F., Bilige, S., Liu, J.G., Li, M.C.: Bright-dark solitons and interaction phenomenon for p-gBKP equation by using bilinear neural network method. Phys. Scr. 96, 025224 (2021)

[38] Hirota, R., Direct Methods in Soliton Theory. Springer, Berlin (1980)

[39] Feng Y.J., Gao Y.T., Li L.Q., Jia, T.T.: Bilinear form and solutions of a (3+1)-dimensional generalized nonlinear evolution equation for the shallow-water waves. Appl. Anal. 378, 1-13 (2019)

[40] Shen Y., Tian B.: Bilinear auto-Bäcklund transformations and soliton solutions of a (3+1)-dimensional generalized nonlinear evolution equation for the shallow-water waves. Appl. Math. Lett. 21, 107301 (2021)

[41] Hirota, R., Satsuma, J.: $N$-soliton solutions of model equation for shallow water waves. J. Phys. Soc. Japan 40(2), 611-612 (1976)

[42] Tan, W., Zhang, W., Zhang, J.: Evolutionary behaviour of breathers and interaction solutions with $M$ solitons for (2+1)-dimensional KdV system. Appl. Math. Lett. 101(C), 106063 (2020)

[43] Tian, Y., Dai, Z.D.: Rogue waves and new multi-wave solutions of the (2+1)-dimensional Ito equation. Z. Naturforsch. A 70(6), 437-443 (2015) 